\title{
Superspace Representations of SU(2,2/N) Superalgebras and Multiplet Shortening
}

\section{S. Ferrara}

Theoretical Physics Division, CERN, Geneva 23, Switzerland

E-mail: 'Sergio.Ferrara@cern.ch'

ABstraCt: We derive massless and massive representations of all $\mathrm{SU}(2,2 / \mathrm{N})$ superalgebras by using superfields defined in "harmonic superspace". This method allows one to easily construct "short superfields" which are relevant in the analysis of the AdS/CFT correspondence.

\section{Introduction}

The study of superconformal algebras has recently attracted renewed interest for their dual role in

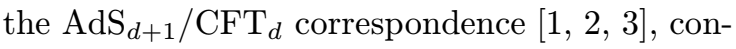
nected to the near-horizon geometry of $d-1$ branes.

A special role is played by 3 -branes since they are related to superconformal invariant quantum Yang-Mills theories. These theories are the only ones exhibiting conformal symmetry both at weak and strong coupling and, in any case, admitting, unlike other types of branes, Yang-Mills fields in the conformal regime.

The bulk and boundary operators in this correspondence are classified by highest weight UIR's

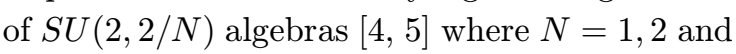
4 in the known examples, since supergravity or superstring theory can admit at most $32(8 N)$ supersymmetries. Nevertheless, in the study of superconformal algebras and their representations different values of $N$ are of interest because they help one to exhibit some general features of short representations, corresponding to conformal operators with protected dimension, but more importantly, because these algebras may be relevant for some generalizations of the known schemes in which more than 32 supersymmetries may be re-

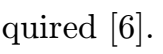

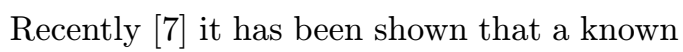
generalization of ordinary superspace, called "harmonic superspace" [is - [10 able to build up, in a rather simple and general manner, all possible composite operators of superconformal invariant gauge theories with $N>$ 1 extended supersymmetry.

Other approaches, like ordinary superspace

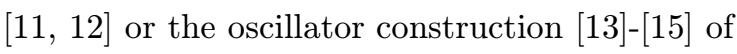
highest weight representations, although in principle possible, are much more complicated to deal with and the complete analysis of all possible shortenings would be unnecessarily difficult.

In fact, the structure of harmonic superspace is powerful enough to allow us to extend the analysis of Ref. [i].] to all $S U(2,2 / N)$ superalgebras with arbitrary $N,{ }^{1}$ although no dynamical theory is known for $N>4$. This report contains

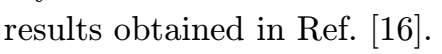

From a mathematical point of view harmonic superspace is an enlarged space where superfields

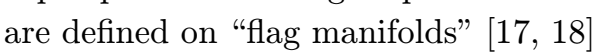

$$
\mathcal{M}=\frac{S U(N)}{S\left(U\left(n_{1}\right) \times \ldots \times U\left(n_{p}\right)\right)},\left(\sum_{k=1}^{p} n_{k}=N\right) .
$$

To study the general case of multiplets it is important [i] to use the choice $n_{k}=1(k=1 \ldots N)$, i.e. where we quotient the group $S U(N)$ by its maximal torus. Then the above manifold is the largest flag manifold with complex dimension $N(N-1) / 2$.

\footnotetext{
${ }^{1}$ For a thorough treatment of the action of superconformal groups on harmonic superspaces see Refs. [1] 17 .
} 
The ultrashort UIR's of $S U(2,2 / N)$ superalgebras described by analytic harmonic superfields depend only on half of the odd coordinates (Grassmann or G-analyticity):

$W^{12 \ldots k}=W^{12 \ldots k}\left(\theta_{k+1}, \theta_{k+2}, \ldots, \theta_{N}, \bar{\theta}^{1}, \bar{\theta}^{2}, \ldots, \bar{\theta}^{k}\right)$.

In addition, they are annihilated by all the "stepup" generators $E_{a}$ in the Cartan decomposition of the Lie algebra of $S U(N)$. In other words, these superfields correspond to highest weight states of $S U(N)$ :

$$
E_{a}|\mathrm{HW}\rangle=0 .
$$

In harmonic superspace this irreducibility condition corresponds to harmonic (or $\mathrm{H}-$ ) analyticity. The crucial point is that the $S U(2,2 / N)$ algebra acting on such states defines a "quasi-primary" superconformal field denoted by

$$
\mathcal{D}\left(\ell, J_{1}, J_{2} ; r ; a_{1}, \ldots, a_{N-1}\right)
$$

where $\ell, J_{1}, J_{2}$ are the conformal dimension and spin of the state, $r$ is the $U(1) R$ charge and $a_{1}, \ldots, a_{N-1}$ are the $S U(N)$ Dynkin labels. We assign the $R$ charge $r_{\theta}=\frac{1}{2}\left(1-\frac{4}{N}\right)$ to the Grassmann coordinates in order to be consistent with the convention that chiral superfields $\Phi(\theta)$ have $l=-r$ for any $N$. This is also the charge which naturally appears in the definition of the $S U(2,2 / N)$ superalgebra [1 $\left.19_{1}^{1}\right]{ }^{2}$

The G- and H-analytic superfields (1..2) have their lowest (scalar) component belonging to the rank $k$ antisymmetric representation of $S U(N)$ $\left(k=1 \ldots\left[\frac{N}{2}\right]\right)$, have $R$ charge $r_{k}=\frac{2 k}{N}-1$ and will be shown to describe "ultrashort" representations of the $S U(2,2 / N)$ superalgebra. If the algebra is interpreted as acting on $\mathrm{AdS}_{5}$, these are the "supersingleton" representations [2] $2 \overline{2}]$. For $k=0$ the superfield is actually "chiral" and in this case the highest weight state may carry a spin label $\left(J_{L}, 0\right)$ with $\ell=1+J_{L}$. The chiral superfield is the supersingleton representation when the top spin is $J_{L}=\frac{N}{2}$. For all other analytic superfields $(k>1)$ the supersingleton will have top $\operatorname{spin} J_{L}=\frac{N}{2}-\frac{k}{2}$.

\footnotetext{
${ }^{2}$ Note that for $N=4, r_{\theta}=0$ and the $r$ quantum number becomes a "central charge" $[20,121$. In this case the analysis of section 2 refers to the $\bar{P} \vec{S} \bar{U}(\overline{2}, 2 / 4)$ algebra for $r=0$ and to the $P U(2,2 / 4)$ algebra for $r \neq 0$.
}

It should be pointed out that the same massless multiplets can be described in terms of or-

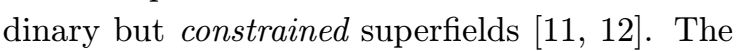
reason why we prefer the harmonic superspace version is the fact that the superfields (1.2) are unconstrained analytic objects. Analyticity is a property which is preserved by multiplication. This will allow us to tensor the above massless UIR's in a very simple way and thus obtain series of short multiplets of $S U(2,2 / N)$. We observe that from the $\mathrm{AdS}_{5}$ point of view, tensoring more than two supersingleton reps produces "massive bulk" reps, while tensoring only two of them produces "massless bulk" reps $\left[\overline{2} \overline{2}_{\mathrm{b}}^{1} \overline{1} \overline{5}\right]$. The latter are the "supercurrent" multiplets discussed in Ref. [1]

\section{Unitarity bounds and shortening of UIR's of $S U(2,2 / N)$}

The unitarity bounds of highest weight UIR's of $S U(2,2 / N)$ have been derived in Refs. [230, 20 , $2 \overline{1}, \overline{4}, \overline{4}$. They correspond to some bounds on

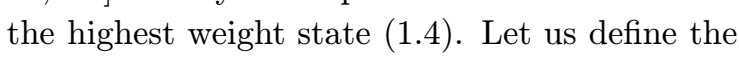
quantities

$$
m_{1}=\sum_{k=1}^{N-1} a_{k}, \quad m=\sum_{k=1}^{N-1}(N-k) a_{k}
$$

and

$$
\begin{aligned}
X\left(J, r, \frac{2 m}{N}\right) & =2+2 J-r+\frac{2 m}{N}, \\
Y\left(r, \frac{2 m}{N}\right) & =-r+\frac{2 m}{N} .
\end{aligned}
$$

Then we have $\left(J_{1}=J_{L}, J_{2}=J_{R}\right)$ :

$$
\begin{aligned}
& \text { A) } \quad \ell \geq X\left(J_{2}, r, \frac{2 m}{N}\right) \geq X\left(J_{1},-r, 2 m_{1}-\frac{2 m}{N}\right) \\
& \left(\text { or } J_{1} \rightarrow J_{2}, r \rightarrow-r, \frac{2 m}{N} \rightarrow 2 m_{1}-\frac{2 m}{N}\right) ; \\
& \text { B) } \quad \ell=Y\left(r, \frac{2 m}{N}\right) \geq X\left(J_{1},-r, 2 m_{1}-\frac{2 m}{N}\right) \\
& \text { (or } \left.J_{1} \rightarrow J_{2}, r \rightarrow-r, \frac{2 m}{N} \rightarrow 2 m_{1}-\frac{2 m}{N}\right) ; \quad \\
& \text { C) } \quad \ell=m_{1}, \quad r=\frac{2 m}{N}-m_{1}, \quad J_{1}=J_{2}=0 .
\end{aligned}
$$

The massless UIR's correspond to B) for $a_{k}=$ $0, \ell=-r=1+J_{L}$ and to $\mathrm{C}$ ) for $\ell=m_{1}=1$, 
$r_{k}=\frac{2 k}{N}-1,1 \leq k \leq\left[\frac{N}{2}\right]$. Note that the two series overlap for $J_{L}=0$ in $\mathrm{B}$ ) and $k=0$ in $\mathrm{C}$ ).

The short multiplets that we shall build in section 4 by tensoring massless multiplets from the C) series in the case of $N=2 n$ for $k=n$ $(r=0)$ will belong to the shortenings in B) and C) obtained for $J_{1}=0$ and $r=0$ :

$$
\begin{aligned}
& \text { B) } \ell=\frac{2 m}{N}, \quad \frac{2 m}{N}-m_{1} \geq 1 \\
& \text { C) } \ell=m_{1}, \quad \frac{2 m}{N}=m_{1}
\end{aligned}
$$

\section{Massless superconformal multiplets}

\subsection{Grassmann analytic superfields}

We consider superfields

$$
W^{i_{1} \ldots i_{k}}\left(x_{\alpha \dot{\alpha}}, \theta_{i}^{\alpha}, \bar{\theta}^{\dot{\alpha} i}\right)
$$

with $k=1, \ldots, n$ (where $n=\left[\frac{N}{2}\right]$ ) totally antisymmetrized indices in the fundamental representation of $S U(N)$. These superfields satisfy the following constraints:

$$
\begin{aligned}
& D_{\alpha}^{(j} W^{\left.i_{1}\right) i_{2} \ldots i_{k}}=0, \\
& \bar{D}_{\dot{\alpha}\{j} W^{\left.i_{1}\right\} i_{2} \ldots i_{k}}=0
\end{aligned}
$$

where () means symmetrization and \{\} means the traceless part. The spinor derivatives algebra is

$$
\left\{D_{\alpha}^{i}, \bar{D}_{\dot{\alpha} j}\right\}=i \delta_{j}^{i} \partial_{\alpha \dot{\alpha}}
$$

with $\partial_{\alpha \dot{\alpha}}=\sigma_{\alpha \dot{\alpha}}^{\mu} \partial_{\mu}$. In the cases $N=2,3,4$ these constraints define the on-shell $N=2$ matter (hyper)multiplet [25in] and the $N=3,4$ on-shell super-Yang-Mills multiplets [2 $\left.\overline{6}_{1}^{\prime}\right]$. Their generalization to arbitrary $N$ has been given in Refs. [1군, $\left.1 \overline{1}_{2}^{\prime}\right]$ where it has also been shown that they describe on-shell massless multiplets.

Our aim in this section is to rewrite the constraints $(\overline{3} . \overline{1}),(\overline{3}) \overline{2})$ in harmonic superspace where they will take the simple form of analyticity conditions. Using this fact we will then be able to construct tensor products of the corresponding multiplets in a very straightforward and easy way (section 4).

The main purpose of introducing harmonics is to be able to covariantly project all the $S U(N)$ indices in (3.1), (3.2) onto a set of $U(1)$ charges. To this end we choose the harmonic coset $S U(N) /(U(1))^{N-1}$ described in terms of harmonic variables $u_{i}^{I}$ and their conjugates $u_{I}^{i}=$ $\left(u_{i}^{I}\right)^{*} .3$ They form an $S U(N)$ matrix where $i$ is an index in the fundamental representation of $S U(N)$ and $I=1, \ldots, N$ is a collection of the $N-1 U(1)$ charges corresponding to the projections of the second index (the harmonic $u_{I}^{i}$ carries charges opposite to those of $u_{i}^{I}$ ). They satisfy the following $S U(N)$ defining conditions:

$$
\begin{aligned}
& u_{i}^{I} u_{J}^{i}=\delta_{J}^{I}, \\
& u_{i}^{I} u_{I}^{j}=\delta_{i}^{j}, \\
& \varepsilon^{i_{1} \ldots i_{N}} u_{i_{1}}^{1} \ldots u_{i_{N}}^{N}=1
\end{aligned}
$$

Now, let us use these harmonic variables to split all the $S U(N)$ indices in the constraints (13.1), (3) into independent $(U(1))^{N-1}$ projections. For example, the projection

$$
W^{12 \ldots k}=W^{i_{1} i_{2} \ldots i_{k}} u_{i_{1}}^{1} u_{i_{2}}^{2} \ldots u_{i_{k}}^{k}
$$

satisfies the constraints

$$
\begin{aligned}
D_{\alpha}^{1} W^{12 \ldots k} & =D_{\alpha}^{2} W^{12 \ldots k}=\ldots= \\
D_{\alpha}^{k} W^{12 \ldots k} & =0 \\
\bar{D}_{\dot{\alpha} k+1} W^{12 \ldots k} & =\bar{D}_{\dot{\alpha} k+2} W^{12 \ldots k}=\ldots= \\
\bar{D}_{\dot{\alpha} N} W^{12 \ldots k} & =0
\end{aligned}
$$

where $D_{\alpha}^{I}=D_{\alpha}^{i} u_{i}^{I}$ and $\bar{D}_{\dot{\alpha} I}=\bar{D}_{\dot{\alpha} i} u_{I}^{i}$. The first of them, eq. (3.81), is a corollary of the commuting nature of the harmonics variables, and the second one, eq. (??), of the unitarity condition $(3 . \overline{4})$. The main achievement in rewriting the constraints $(3.1),(3.2)$ in this new form is that they can be explicitly solved by going to an appropriate G-analytic basis in superspace:

$$
\begin{aligned}
& x_{A}^{\alpha \dot{\alpha}}=x^{\alpha \dot{\alpha}}+i\left(\theta_{1}^{\alpha} \bar{\theta}^{1 \dot{\alpha}}+\ldots+\theta_{k}^{\alpha} \bar{\theta}^{k \dot{\alpha}}\right. \\
& \left.-\theta_{k+1}^{\alpha} \bar{\theta}^{k+1 \dot{\alpha}}-\ldots-\theta_{N}^{\alpha} \bar{\theta}^{N \dot{\alpha}}\right) \\
& \theta_{I}^{\alpha}=\theta_{i}^{\alpha} u_{I}^{i}, \quad \bar{\theta}^{\dot{\alpha} I}=\bar{\theta}^{\dot{\alpha} i} u_{i}^{I} .
\end{aligned}
$$

In this basis $W^{12 \ldots k}$ becomes an unconstrained function of $k \bar{\theta}$ 's and $N-k \theta$ 's:

$W^{12 \ldots k}=W^{12 \ldots k}\left(x_{A}, \theta_{k+1}, \ldots, \theta_{N}, \bar{\theta}^{1}, \ldots, \bar{\theta}^{k}, u\right)$.

\footnotetext{
${ }^{3}$ The harmonic notation used here differs from the original one of Refs. troduced in Ref. [10] for the case $N=3$ and in Refs. [1] for general $N$.
} 
Altogether it depends on half the number of the odd variables of $N$-extended superspace and for this reason we call it Grassmann (or G-) analytic. We recall that the notion of Grassmann analyticity was first introduced in Ref. [27], still in the context of ordinary superspace. In $N=2$ harmonic superspace [i] this notion became $S U(2)$ covariant. The generalization to $N=3$ was given in Ref. [9in and later on to general $N$ in Refs. [1] (under the name of " $(N, p, q)$ superspace").

The massless conformal multiplets describe the ordinary massless UIR's of the super Poincaré group obtained earlier by the Wigner method of induced representations (see, for instance, Ref. $\left[2 \overline{8}^{\prime}\right]$ ). The self-conjugate $N=8$ multiplet was obtained by the oscillator method in Ref. [2 $\left.29_{1}^{\prime}\right]$.

\subsection{Harmonic analyticity as $S U(N)$ irreducibility}

It is important to realize that a G-analytic superfield is an $S U(N)$ covariant object only because it depends on the harmonic variables. In order to recover the original harmonic-independent but constrained superfield $W^{i_{1} i_{2} \ldots i_{k}}(x, \theta, \bar{\theta})(\overline{3} \cdot \overline{1})$, $(3.2)$ we need to impose differential conditions involving the harmonic variables. The harmonic derivatives are made out of the operators

$$
\partial_{J}^{I}=u_{i}^{I} \frac{\partial}{\partial u_{i}^{J}}-u_{J}^{i} \frac{\partial}{\partial u_{I}^{i}}
$$

which respect the defining relations $(3.4),(3.5)$. These derivatives act on the harmonics as follows:

$$
\partial_{J}^{I} u_{i}^{K}=\delta_{J}^{K} u_{i}^{I}, \quad \partial_{J}^{I} u_{K}^{i}=-\delta_{K}^{I} u_{J}^{i} .
$$

The diagonal ones $\partial_{I}^{I}$ count the $U(1)$ charges,

$$
\partial_{I}^{I} u_{i}^{I}=u_{i}^{I}, \quad \partial_{I}^{I} u_{I}^{i}=-u_{I}^{i} .
$$

The relation (3.6i) implies that the charge operators $\partial_{I}^{I}$ are not independent,

$$
\sum_{I=1}^{N} \partial_{I}^{I}=0
$$

(this reflects the fact that we are considering $S U(N)$ and not $U(N))$.

A basic assumption in our approach to the harmonic coset $S U(N) / U(1)^{N-1}$ is that any harmonic function is homogeneous under the action of $U(1)^{N-1}$, i.e., it is an eigenfunction of the charge operators $\partial_{I}^{I}$,

$$
\begin{aligned}
& \partial_{I}^{I} f_{L_{1} \ldots L_{r}}^{K_{1} \ldots K_{q}}(u)=\left(\delta_{I}^{K_{1}}+\ldots+\delta_{I}^{K_{q}}-\delta_{L_{1}}^{I}-\ldots\right. \\
& \left.-\delta_{L_{r}}^{I}\right) f_{L_{1} \ldots L_{r}}^{K_{1} \ldots K_{q}}(u)
\end{aligned}
$$

(note that the charges $K_{1} \ldots K_{q} ; L_{1} \ldots L_{r}$ are not necessarily all different). Thus it effectively depends on the $\left(N^{2}-1\right)-(N-1)=N(N-1)$ real coordinates of the coset $S U(N) / U(1)^{N-1}$. Then the actual harmonic derivatives on the coset are the $N(N-1) / 2$ complex derivatives $\partial_{J}^{I}, I<J$ (or their conjugates $\partial_{J}^{I}, I>J$ ).

The set of $N^{2}-1$ derivatives $\partial_{J}^{I}$ (taking into account the linear dependence $\left.\left({ }^{3} . \overline{1} \overline{5}_{1}\right)\right)$ form the algebra of $S U(N)$ :

$$
\left[\partial_{J}^{I}, \partial_{L}^{K}\right]=\delta_{J}^{K} \partial_{L}^{I}-\delta_{L}^{I} \partial_{J}^{K}
$$

The Cartan decomposition of this algebra $L^{+}+$ $L^{0}+L^{-}$is given by the sets

$$
\begin{aligned}
& L^{+}=\left\{\partial_{J}^{I}, I<J\right\}, \quad L^{0}=\left\{\partial_{I}^{I}, \sum_{I=1}^{N} \partial_{I}^{I}=0\right\}, \\
& L^{-}=\left\{\partial_{J}^{I}, I>J\right\} .
\end{aligned}
$$

It becomes clear that imposing the harmonic conditions

$$
\partial_{J}^{I} f_{L_{1} \ldots L_{r}}^{K_{1} \ldots K_{q}}(u)=0, \quad I<J
$$

on a harmonic function with a given set of charges $K_{1} \ldots K_{q} ; L_{1} \ldots L_{r}$ defines the highest weight of an $S U(N)$ irrep. In other words, the harmonic expansion of such a function contains only one irrep which is determined by the combination of charges $K_{1} \ldots K_{q} ; L_{1} \ldots L_{r}$. In fact, not all of the derivatives $\partial_{J}^{I}, I<J$ are independent, as follows from the algebra $\left(\bar{\beta} \cdot \overline{1} \overline{7}_{1}\right)$. The independent set consists of the $N-1$ derivatives

$$
\partial_{2}^{1}, \partial_{3}^{2}, \ldots, \partial_{N}^{N-1}
$$

corresponding to the simple roots of $S U(N)$. Then the $S U(N)$ defining constraint $(\overline{3} . \overline{1} \bar{q})$ is equivalent to

$$
\left(\partial_{2}^{1}, \partial_{3}^{2}, \ldots, \partial_{N}^{N-1}\right) f_{L_{1} \ldots L_{r}}^{K_{1} \ldots K_{q}}(u)=0 .
$$

The coset $S U(N) / U(1)^{N-1}$ can be parametrized by $N(N-1) / 2$ complex coordinates. In this case 
the constraints $(\overline{3} . \overline{1} \overline{9})$ take the form of covariant (in the sense of Cartan) Cauchy-Riemann analyticity conditions. For this reason we call the set of constraints $(\bar{\beta} . \overline{1} \bar{g})$ (or the equivalent set $(\overline{3} . \overline{2} \overline{1})$ ) harmonic (H-)analyticity conditions. The above argument shows that $\mathrm{H}$-analyticity is equivalent to defining a highest weight of $S U(N)$, i.e. it is the $S U(N)$ irreducibility condition on the harmonic functions.

As an example, take $N=2$ and the function $f^{1}(u)$ subject to the constraint

$$
\partial_{2}^{1} f^{1}(u)=0 \Rightarrow f^{1}(u)=f^{i} u_{i}^{1} .
$$

So, the harmonic function is reduced to a doublet of $S U(2)$. Similarly, for $N=4$ the function $f^{12}(u)$ is reduced to the $\underline{6}$ of $S U(4)$. Indeed, the constraints $\partial_{3}^{2} f^{12}(u)=\partial_{4}^{3} f^{12}(u)=0$ ensure that $f^{12}(u)$ depends on $u^{1}, u^{2}$ only, $f^{12}(u)=$ $f^{i j} u_{i}^{1} u_{j}^{2}$. Then the constraint $\partial_{2}^{1} f^{12}(u)=f^{i j} u_{i}^{1} u_{j}^{1}$ $=0$ implies $f^{i j}=-f^{j i}$.

In the G-analytic basis $(\overline{13} . \overline{10})$ the harmonic derivatives become covariant $D_{J}^{I}$. In particular, the derivatives

$$
\begin{aligned}
D_{J}^{I} & =\partial_{J}^{I}-i \theta_{J}^{\alpha} \bar{\theta}^{I} \dot{\alpha} \partial_{\alpha \dot{\alpha}}-\theta_{J} \partial^{I}+\bar{\theta}^{I} \bar{\partial}_{J} \\
I & =1, \ldots, k, J=k+1, \ldots, N
\end{aligned}
$$

acquire space-time derivative terms. The $S U(N)$ commutation relations among the $D_{J}^{I}$ are not affected by the change of basis. The same is true for the commutation relations of the $D_{J}^{I}$ with the spinor derivatives:

$\left[D_{J}^{I}, D_{\alpha}^{K}\right]=\delta_{J}^{K} D_{\alpha}^{I}, \quad\left[D_{J}^{I}, \bar{D}_{\dot{\alpha} K}\right]=-\delta_{K}^{I} \bar{D}_{\dot{\alpha} J}$

Using these relations one can see that the $\mathrm{H}$ analyticity conditions

$$
D_{J}^{I} W^{12 \ldots k}=0, \quad I<J
$$

or the equivalent set

$$
\left(D_{2}^{1}, D_{3}^{2}, \ldots, D_{N}^{N-1}\right) W^{12 \ldots k}=0
$$

are compatible with the G-analyticity ones (??).

\subsection{Analyticity and massless multiplets: "Singletons"}

The constraints of H-analyticity $(\overline{3} \cdot \overline{2} \overline{5})$ combined with those of G-analyticity (??) have important implications for the components of the superfield. First of all, they make each component an irrep of $S U(N)$. Take, for example, the first component

$$
\phi^{12 \ldots k}(x, u)=\left.W^{12 \ldots k}\right|_{0}
$$

where $\left.\right|_{0}$ means $\theta=\bar{\theta}=0$. The constraints $\partial_{I+1}^{I} \phi^{12 \ldots k}(x, u)=0, I=k, \ldots, N$ imply that $\phi^{12 \ldots k}(x, u)$ takes the form

$$
\phi^{12 \ldots k}(x, u)=\phi^{i_{1} i_{2} \ldots i_{k}}(x) u_{i_{1}}^{1} u_{i_{2}}^{2} \ldots u_{i_{k}}^{k} .
$$

This is a rank $k$ tensor without any symmetry, i.e. a reducible representation of $S U(N)$. Further, the constraint, e.g.,

$$
\begin{gathered}
\partial_{2}^{1} \phi^{123 \ldots k}(x, u)=\phi^{113 \ldots k}(x, u)= \\
\phi^{i_{1} i_{2} i_{3} \ldots i_{k}} u_{i_{1}}^{1} u_{i_{2}}^{1} u_{i_{3}}^{3} \ldots u_{i_{k}}^{n}=0
\end{gathered}
$$

removes the symmetric part in the first two indices. Similarly, the remaining constraints (3. remove all the symmetrizations and we find the totally antisymmetric rank $k$ irrep of $S U(N)$.

Another example are the spinor components

$$
\begin{aligned}
& \chi_{\alpha}^{12 \ldots k k+1}(x, u)=\left.D_{\alpha}^{k+1} W^{12 \ldots k}\right|_{0}, \\
& \bar{\psi}_{\dot{\alpha}}^{23 \ldots k}(x, u)=\left.\bar{D}_{1 \dot{\alpha}} W^{12 \ldots k}\right|_{0} .
\end{aligned}
$$

The same harmonic argument shows that these are harmonic projections of the totally antisymmetric components $\chi_{\alpha}^{\left[i_{1} i_{2} \ldots i_{k+1}\right]}(x)$ and $\bar{\psi}_{\dot{\alpha}}^{\left[i_{2} i_{3} \ldots i_{k}\right]}(x)$.

Further important constraints occur at the level of 2 or more $\theta$ 's:

$$
\begin{aligned}
& D^{I \alpha} D_{\alpha}^{J} W^{12 \ldots k}=0, I, J=k+1, \ldots, N, \\
& \bar{D}_{I \dot{\alpha}} \bar{D}_{J}^{\dot{\alpha}} W^{12 \ldots k}=0, I, J=1, \ldots, k .
\end{aligned}
$$

The easiest way to see this is to hit the defining constraint (3.1) with $D^{k \alpha}$ and then project with harmonics.

The constraints $\left(\overline{3} . \overline{2} \overline{9^{\prime}}\right),\left(\overline{3} . \overline{3} 0^{\prime \prime}\right)$ imply that the components of the type

$$
\begin{aligned}
& \chi_{\left(\alpha_{1} \ldots \alpha_{p}\right)}^{1 \ldots k+p}=\left.D_{\alpha_{1}}^{k+1} \ldots D_{\alpha_{p}}^{k+p} W^{12 \ldots k}\right|_{0}, p \leq N-k \\
& \bar{\psi}_{\left(\dot{\alpha}_{1} \ldots \dot{\alpha}_{p}\right)}^{p+1 \ldots k}=\bar{D}_{\left.1 \dot{\alpha}_{1} \ldots \bar{D}_{p \dot{\alpha}_{p}} W^{12 \ldots k}\right|_{0}, \quad p \leq k}
\end{aligned}
$$


are totally symmetric in their spinor indices, i.e. they carry $\operatorname{spin}(p / 2,0)$ or $(0, p / 2)$, correspondingly. Among them one finds the

$$
\begin{aligned}
& \text { top spin }\left(\frac{N}{2}-\frac{k}{2}, 0\right): \quad \chi_{\left(\alpha_{1} \ldots \alpha_{N-k}\right)}= \\
& \left.D_{\alpha_{1}}^{k+1} \ldots D_{\alpha_{N-k}}^{N} W^{12 \ldots k}\right|_{0}
\end{aligned}
$$

which is also an $S U(N)$ singlet. Note that in the case $N=2 n, k=n$ the top spin occurs both as $(n / 2,0)$ and $(0, n / 2)$ (we call this a "selfconjugate" multiplet). Moreover, if $N=4 n$ and $k=2 n$ one can impose a reality condition on the superfield $W^{12 \ldots 2 n}$ which implies, in particular, that

$$
\chi_{\left(\alpha_{1} \ldots \alpha_{2 n}\right)}=\left(\psi_{\left(\dot{\alpha}_{1} \ldots \dot{\alpha}_{2 n}\right)}\right)^{*} .
$$

Next, one can show that all the components of the type $(\overline{3} . \overline{3} \overline{1}),\left(\underline{3} . \overline{3} 2^{i}\right)$ satisfy massless field equations. Indeed, from the constraint ( $\overline{3} . \overline{2} \overline{9})$ and from G-analyticity it follows that

$$
\begin{aligned}
0 & =\bar{D}_{k+1 \dot{\beta}} D^{k+1 \alpha_{1}} D_{\alpha_{1}}^{k+1} \ldots D_{\alpha_{p}}^{k+p} W^{12 \ldots k} \\
& =2 i \partial_{\dot{\beta}}^{\alpha_{1}} D_{\alpha_{1}}^{k+1} \ldots D_{\alpha_{p}}^{k+p} W^{12 \ldots k} \\
& \Rightarrow \partial_{\dot{\beta}}^{\alpha_{1}} \chi_{\left(\alpha_{1} \ldots \alpha_{p}\right)}^{1 \ldots k+p}=0
\end{aligned}
$$

and similarly for $\bar{\psi}_{\left(\dot{\alpha}_{1} \ldots \dot{\alpha}_{p}\right)}^{p+1 \ldots k}$. The leading scalar

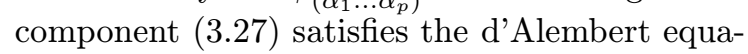
tion:

$$
\begin{aligned}
0 & =\left(D^{1}\right)^{2}\left(\bar{D}_{1}\right)^{2} W^{12 \ldots k}=4 \square W^{12 \ldots k} \\
& \Rightarrow \square \phi^{12 \ldots k}=0 .
\end{aligned}
$$

Finally, all the components of mixed type,

$$
\begin{aligned}
& f_{\dot{\alpha}_{1} \ldots \dot{\alpha}_{p} \alpha_{1} \ldots \alpha_{q}}^{p+1 \ldots k+q} \\
& =\left.\bar{D}_{1 \dot{\alpha}_{1}} \ldots \bar{D}_{p \dot{\alpha}_{p}} D_{\alpha_{1}}^{k+1} \ldots D_{\alpha_{q}}^{k+q} W^{12 \ldots k}\right|_{0} \\
& p \leq k, q \leq N-k
\end{aligned}
$$

are expressed in terms of the space-time derivatives of lower components. Indeed,

$$
\begin{aligned}
& D_{k+q}^{1} f_{\dot{\alpha}_{1} \ldots \dot{\alpha}_{p} \alpha_{1} \ldots \alpha_{q}}^{p+1 \ldots k+q} \\
& =-\left.\bar{D}_{k+q \dot{\alpha}_{1}} \bar{D}_{2 \dot{\alpha}_{2}} \ldots D_{\alpha_{q}}^{k+q} W^{12 \ldots k}\right|_{0} \\
& =\left.(-1)^{p+q-1} i \partial_{\dot{\alpha}_{1} \alpha_{q}} \bar{D}_{2 \dot{\alpha}_{2}} \ldots D_{\alpha_{q-1}}^{k+q-1} W^{12 \ldots k}\right|_{0} \\
& \Rightarrow f_{\dot{\alpha}_{1} \ldots \dot{\alpha}_{p} \alpha_{1} \ldots \alpha_{q}}^{p+1 \ldots k+q-1} 1 \\
& =(-1)^{p+q-1} i \partial_{\dot{\alpha}_{1} \alpha_{q}} g_{\dot{\alpha}_{2} \ldots \dot{\alpha}_{p} \alpha_{1} \ldots \alpha_{q-1}}^{1 p+1 \ldots k+q-1}
\end{aligned}
$$

To summarize, the superfield $W^{12 \ldots k}$ subject to the constraints of G- and H-analyticity has the following component content (the derivative terms are not shown):

$$
\begin{aligned}
& W^{12 \ldots k}=\phi^{12 \ldots k} \\
& +\bar{\theta}_{\dot{\alpha}}^{1} \bar{\psi}^{\dot{\alpha} 23 \ldots k}+\ldots+\bar{\theta}_{\dot{\alpha}}^{k} \bar{\psi}^{\dot{\alpha} 12 \ldots k-1} \\
& +\theta_{k+1}^{\alpha} \chi_{\alpha}^{1 \ldots k k+1}+\ldots+\theta_{N}^{\alpha} \chi_{\alpha}^{1 \ldots k N} \\
& +\bar{\theta}_{\dot{\alpha}}^{1} \bar{\theta}_{\dot{\beta}}^{2} \bar{\psi}^{(\dot{\alpha} \dot{\beta}) 3 \ldots k}+\ldots+\bar{\theta}_{\dot{\alpha}}^{k-1} \bar{\theta}_{\dot{\beta}}^{k} \bar{\psi}^{(\dot{\alpha} \dot{\beta})} 1 \ldots k-2 \\
& +\theta_{k+1}^{\alpha} \theta_{k+2}^{\beta} \chi_{(\alpha \beta)}^{1 \ldots k k+1 k+2}+ \\
& \ldots+\theta_{N-1}^{\alpha} \theta_{N}^{\beta} \chi_{(\alpha \beta)}^{1 \ldots k N-1 N} \ldots \\
& +\bar{\theta}_{\dot{\alpha}_{1}}^{1} \ldots \bar{\theta}_{\dot{\alpha}_{k}}^{k} \bar{\psi}^{\left(\dot{\alpha}_{1} \ldots \dot{\alpha}_{k}\right)} \\
& +\theta_{k+1}^{\alpha_{1}} \ldots \theta_{N}^{\alpha_{N-k}} \chi_{\left(\alpha_{1} \ldots \alpha_{N-k}\right)}
\end{aligned}
$$

where all the fields belong to totally antisymmetric irreps of $S U(N)$ and satisfy the massless field equations

$$
\begin{aligned}
& \square \phi^{\left[i_{1} \ldots i_{k}\right]}=0, \\
& \partial^{\beta \dot{\alpha}_{1}} \bar{\psi}_{\left(\dot{\alpha}_{1} \ldots \dot{\alpha}_{p}\right)}^{\left[i_{1} \ldots i_{k-p}\right]}=0, \quad 1 \leq p \leq k \\
& \partial^{\alpha_{1} \dot{\beta}} \chi_{\left(\alpha_{1} \ldots \alpha_{p}\right)}^{\left[i_{1} \ldots i_{p}\right]}=0, \quad 1 \leq p \leq N-k
\end{aligned}
$$

This is the content of an $N$-extended superconformal multiplet of the $\mathrm{C}$ ) series of section 2 . It is characterized by the $S U(N)$ irrep of the first component (described by the Young tableau $\left.m_{1}=\ldots=m_{k}=1, m_{k+1}=\ldots=m_{N-1}=0\right)$, by its $R$ charge

$$
r_{k}=\frac{2 k}{N}-1
$$

and conformal dimension $\ell=1$ and by the top $\operatorname{spin} J_{\text {top }}=\left(\frac{N}{2}-\frac{k}{2}, 0\right)$.

\subsection{Chiral superfields}

The G-analytic superfields considered above contain at least one $\bar{\theta}$. The case of "extreme" Ganalyticity will be the absence of any $\bar{\theta}$ 's. These are the well-known chiral superfields [300 ing the constraint

$$
\bar{D}_{i \dot{\alpha}} W=0 \quad \Rightarrow \quad W=W\left(x_{L}^{\alpha \dot{\alpha}}, \theta_{i}^{\alpha}\right)
$$

where

$$
x_{L}^{\alpha \dot{\alpha}}=x^{\alpha \dot{\alpha}}-i \theta_{i}^{\alpha} \bar{\theta}^{i \dot{\alpha}} .
$$

Note that in this case we do not need harmonic variables, since G-analyticity involves a subset of odd coordinates forming an entire irrep of $S U(N)$, and not a set of $U(1)$ projections. Consequently, 
in order to put such a superfield on shell, we cannot use $\mathrm{H}$-analyticity but need to impose a new type of constraint:

$$
D^{\alpha i} D_{\alpha}^{j} W=0
$$

The resulting components are multispinors of the same chirality (cf. eq. $(\underline{3} \cdot \overline{3} \cdot \bar{j}))$ :

$$
\begin{aligned}
W & =\phi+\theta_{i}^{\alpha} \chi_{\alpha}^{i} \\
& +\ldots+\theta_{i_{1}}^{\alpha_{1}} \ldots \theta_{i_{n}}^{\alpha_{n}} \chi_{\left(\alpha_{1} \ldots \alpha_{n}\right)}^{\left[i_{1} \ldots i_{n}\right]}+\ldots+(\theta)^{2 N} \chi
\end{aligned}
$$

satisfying massless field equations. The tops spin is $\left(\frac{N}{2}, 0\right)$.

The chiral superfields above are scalar, but there exist conformally covariant chiral superfields with an arbitrary $\left(J_{L}, 0\right)$ index of the highest weight: $W_{\alpha_{1} \ldots \alpha_{2 J}}$. In this case the masslessness condition is [1] $\left.{ }_{1}^{2}\right] D^{\alpha_{1} i} W_{\alpha_{1} \ldots \alpha_{2 J_{L}}}=0$.

\section{Short superconformal multiplets: bulk " massless" and "massive" states}

In this section we shall concentrate on the case $N=2 n$ for reasons of simplicity. The analytic superfield $W^{12 \ldots n}\left(\theta_{n+1}, \ldots, \theta_{2 n}, \bar{\theta}^{1}, \ldots, \bar{\theta}^{n}\right)$ describes a superconformal multiplet characterized by the Young tableau $m_{1}=\ldots=m_{n}=1$, $m_{n+1}=\ldots=m_{2 n-1}=0$ of its first component (a Lorentz scalar), by its dimension $\ell=1$ and $R$ charge $r=0$ (see $(3 . \overline{4} \overline{1})$ ). Now we shall use this multiplet as a building block for constructing other "short" superconformal multiplets.

The building block $W^{12 \ldots n}$ can be equivalently rewritten by choosing different harmonic projections of its $S U(N)$ indices and, consequently, different sets of G-analyticity constraints. This amounts to superfields of the type

$$
W^{I_{1} I_{2} \ldots I_{n}}\left(\theta_{J_{1}}, \ldots, \theta_{J_{n}}, \bar{\theta}^{I_{1}}, \ldots, \bar{\theta}^{I_{n}}\right)
$$

where $I_{1}, \ldots, I_{n}$ and $J_{1}, \ldots, J_{n}$ are two complementary sets of $n$ indices. Each of these superfields depends on $2 N=4 n$ Grassmann variables, i.e. half of the total number of $4 N=8 n$. This is the minimal size of a G-analytic superspace, so we can say that the $W$ 's are the "shortest" superfields (superconformal multiplets). Another characteristic of these $W^{\prime} \mathrm{s}$ is the absence of $R$ charges.

The idea now is to start multiplying different species of the $W$ 's of the type (4.11) in order to obtain composite objects depending on various numbers of odd variables. The sets $I_{1}, \ldots, I_{n}$ can be chosen in $(2 n) ! /(n !)^{2}$ different ways. However, we do not need consider all of them. The following choice of $W$ 's and of the order of multiplication covers all possible intermediate types of G-analyticity:

$$
A\left(p_{1}, p_{2}, \ldots, p_{2 n-1}\right)=
$$

$$
\begin{aligned}
& \quad\left[W^{1 \ldots n}\left(\theta_{n+1 \ldots 2 n} \bar{\theta}^{1 \ldots n}\right)\right]^{p_{1}+\ldots+p_{2 n-1}} \\
& \times\left[W ^ { 1 \ldots n - 1 n + 1 } \left(\theta_{\underline{n} n+2 \ldots 2 n}\right.\right. \\
& \left.\left.\quad \times \bar{\theta}^{1 \ldots n-1} \underline{n+1}\right)\right]^{p_{2}+\ldots+p_{2 n-1}} \\
& \times\left[W ^ { 1 \ldots n - 1 n + 2 } \left(\theta_{n n+1} n+3 \ldots 2 n\right.\right. \\
& \left.\left.\quad \times \bar{\theta}^{1 \ldots n-1} \underline{n+2}\right)\right]^{p_{3}+\ldots+p_{2 n-1}} \\
& \ldots \\
& \times\left[W ^ { 1 \ldots n - 1 2 n - 1 } \left(\theta_{n \ldots 2 n-22 n}\right.\right. \\
& \left.\left.\quad \times \bar{\theta}^{1 \ldots n-1} \underline{2 n-1}\right)\right]^{p_{n}+\ldots+p_{2 n-1}} \\
& \times \\
& \quad\left[W ^ { 1 \ldots n - 2 n n + 1 } \left(\theta_{n-1}{ }^{n+2 \ldots 2 n}\right.\right. \\
& \left.\left.\quad \times \bar{\theta}^{1 \ldots n-2 n n+1}\right)\right]^{p_{n+1}+\ldots+p_{2 n-1}} \\
& \times\left[W ^ { 1 \ldots n - 3 n - 1 n n + 1 } \left(\theta_{\underline{n-2}} n+2 \ldots 2 n\right.\right. \\
& \left.\left.\quad \times \bar{\theta}^{1 \ldots n-3 n-1 n n+1}\right)\right]^{p_{n+2}+\ldots+p_{2 n-1}} \\
& \ldots \\
& \times\left[W^{13 \ldots n+1}\left(\theta_{\underline{2} n+2 \ldots 2 n} \bar{\theta}^{13 \ldots n+1}\right)\right]^{p_{2 n-2}+p_{2 n-1}} \\
& \times\left[W^{23 \ldots n+1}\left(\theta_{\underline{1} n+2 \ldots 2 n} \bar{\theta}^{23 \ldots n+1}\right)\right]^{p_{2 n-1}} . \quad(4.2)
\end{aligned}
$$

The power $\sum_{r=k}^{2 n-1} p_{r}$ of the $k$-th $W$ is chosen in such a way that each new $p_{r}$ corresponds to bringing in a new type of $W$. As a result, at each step a new $\theta$ or $\bar{\theta}$ appears (they are underlined in $(4.2))$, thus adding new odd dimensions to the Ganalytic superspace. The only exception of this rule is the second step at which both a new $\theta$ and a new $\bar{\theta}$ appear. So, the series (4.2) covers all possible subspaces with $4 n, 4 n+4,4 n+6, \ldots, 8 n-2$ odd coordinates (notice once again the missing subspace with $4 n+2$ odd coordinates). In this sense we can say that the G-analytic superfield $A\left(p_{1}, p_{2}, \ldots, p_{2 n-1}\right)$ realizes a "short" superconformal multiplet.

The superfield $A\left(p_{1}, p_{2}, \ldots, p_{2 n-1}\right)$ should be submitted to the same $\mathrm{H}$-analyticity constraints 
as one would impose on $W^{1 \ldots n}$ alone,

$$
\begin{aligned}
& D_{I+1}^{I} A\left(p_{1}, p_{2}, \ldots, p_{2 n-1}\right)=0 \\
& I=1,2, \ldots, 2 n-1 .
\end{aligned}
$$

This is clearly compatible with G-analyticity since the conditions on a generic $A\left(p_{1}, p_{2}, \ldots, p_{2 n-1}\right)$ form a subset of these on $W^{1 \ldots n}$. As before, $\mathrm{H}$ analyticity makes $A\left(p_{1}, p_{2}, \ldots, p_{2 n-1}\right)$ irreducible under $S U(N)$. Here is the structure of Young tableau which corresponds to the first (scalar) component of this superfield (and characterizes the supermultiplet as a whole):

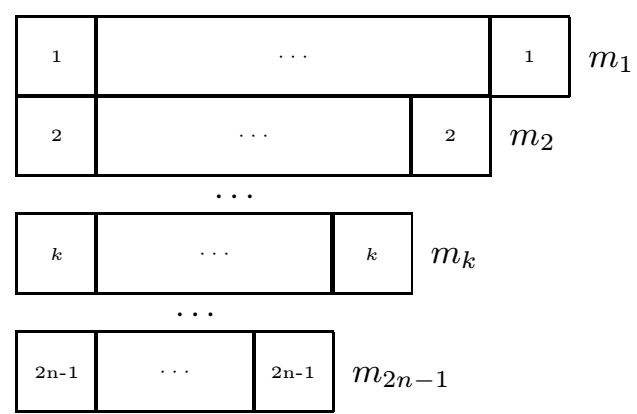

The top row is filled with indices projected with $u_{i}^{1}$ (hence the symmetrization among them), the second row - with $u_{i}^{2}$, etc. The harmonic conditions $\left(\begin{array}{l}-\overline{4} \\ -3\end{array}\right)$ remove all the symmetrizations among indices belonging to different projections (rows). By counting the number of occurrences of the projection 1 in ('s.2.

$$
m_{1}=\ell-p_{2 n-1}
$$

where $\ell$ is the total number of $W$ 's (equal to the dimension of the superfield $A$, since $\ell_{W}=1$ ). Another simple counting shows the relation

$$
\sum_{k=1}^{2 n-1} m_{k}=n \ell=\frac{N}{2} \ell .
$$

If the last $W$ in (4.3i) is not present there is an additional relation among the Young tableau labels:

$$
p_{2 n-1}=0 \quad \Rightarrow \quad m_{1}=\frac{2}{N} \sum_{k=1}^{2 n-1} m_{k} .
$$

Finally, introducing the Dynkin labels $\left[a_{1}, \ldots\right.$, $\left.a_{2 n-1}\right]$ where $a_{1}=m_{2 n-1}$ and $a_{k}=m_{2 n-k+1}-$ $m_{2 n-k}$ for $k \geq 2$, we find

$$
\begin{aligned}
& a_{1}=\sum_{k=n}^{2 n-1} p_{k}, \\
& a_{2}=p_{n-1}, \quad \ldots, \quad a_{n-2}=p_{3}, \\
& a_{n-1}=p_{2}+\sum_{k=n+1}^{2 n-1}(k-n) p_{k}, \\
& a_{n}=p_{1}, \\
& a_{n+1}=(n-2) \sum_{k=n+1}^{2 n-1} p_{k}+\sum_{k=2}^{n}(k-1) p_{k}, \\
& a_{n+2}=p_{n+1}, \quad \ldots, \quad a_{2 n-1}=p_{2 n-2} .
\end{aligned}
$$

\section{Conclusion}

In this paper we studied representations of fourdimensional superconformal algebras with an arbitrary number of supersymmetries.

This analysis also provides the classification of short multiplets of superalgebras on $\mathrm{AdS}_{5}$ and in particular "massless" and "massive" fields in anti-de Sitter geometries, in terms of boundary "composite" operatprs

\section{Acknowledgements}

This work has been supported in part by the European Commission TMR programme ERBFMRXCT96-0045 (Laboratori Nazionali di Frascati, INFN) and by DOE grant DE-FG03-91ER40662, Task C.

\section{References}

[1] J.M. Maldacena, Adv. Theor. Math. Phys. 2 (1998) 231, hep-th/9705104.

[2] S.S. Gubser, I.R. Klebanov and A.M. Polyakov, Phys. Lett. B428 (1998) 105, hep-th/9802109.

[3] E. Witten, Adv. Theor. Math. Phys. 2 (1998) 253 , hep-th/9802150.

[4] S. Ferrara, C. Fronsdal and A. Zaffaroni, Nucl. Phys. B532 (1998) 153, hep-th/9802203; S. Ferrara and C. Fronsdal, Phys. Lett. B433 (1998) 19.

[5] L. Andrianopoli and S. Ferrara, Phys. Lett. B430 (1998) 248, hep-th/9803171. 
[6] I. Bars, Phys. Lett. B403 (1997) 257; I. Bars, C. Deludiman, O. Andreev, Phys. Rev. D58 (1998) 066004; I. Bars, Phys. Rev. D59 (1999) 045019.

[7] L. Andrianopoli, S. Ferrara, E. Sokatchev and B. Zupnik Shortening of primary operators in $\mathrm{N}$ extended $\mathrm{SCFT}_{4}$ and harmonic-superspace analyticity, hep-th/9912007.

[8] A. Galperin, E. Ivanov, S. Kalitzin, V. Ogievetsky and E. Sokatchev, Class. Quant. Grav. 1 (1984) 469; E. Ivanov, S. Kalitsyn, Nguyen Ai Viet and V. Ogievetsky, J. Phys. A18 (1985) 3433.

[9] A. Galperin, E. Ivanov, S. Kalitzin, V. Ogievetsky and E. Sokatchev, Class. Quant. Grav. 2 (1985) 155; R. Kallosh, Pis'ma ZHETF, 41 (1985) 172.

[10] A. Galperin, E. Ivanov and V. Ogievetsky, Sov. J. Nucl. Phys. 46 (1987) 543.

[11] W. Siegel, Nucl. Phys. B177 (1981) 325.

[12] P. Howe, K.S. Stelle and P.K. Townsend, Nucl. Phys. B191 (1981) 445; Nucl. Phys. $\mathbf{B 1 9 2}$ (1981) 332.

[13] I. Bars and M. Gunaydin, Commun. Math. Phys. 91 (1983) 31; M. Gunaydin, J. Math. Phys. 29 (1988) 1275.

[14] M. Gunaydin and N. Marcus, Class. Quantum Grav. 2 (1985) L11.

[15] M. Gunaydin, D. Minic and M. Zagermann, Nucl. Phys. B534 (1998) 96, hep-th/9806042; M. Gunaydin, D. Minic and M. Zagermann, Nucl. Phys. B544 (1999) 737, hep-th/9810226.

[16] S. Ferrara and E. Sokatchev, hep-th/0012168.

[17] G.G. Hartwell and P.S. Howe, Int. J. Mod. Phys. A10 (1995) 3901, hep-th/9412147; Class. Quant. Grav. 12 (1995) 1823.

[18] A.W. Knapp, "Representation theory of semisimple groups", Princeton Universitary Press, 1986.

[19] S. Ferrara, M. Kaku, P.K. Townsend and P. van Nieuwenhuizen, Nucl. Phys. B129 (1977) 125.

[20] V. K. Dobrev and V. B. Petkova, Phys. Lett. B162 (1985) 127; Fortschr. Phys. 35 (1987) 7, 537.

[21] B. Binegar, Phys. Rev. D34 (1986) 525.

[22] M. Flato and C. Fronsdal, Lett. Math. Phys. 2 (1978) 421; Phys. Lett. 97B (1980) 236; J. Math. Phys. 22 (1981) 1100; Phys. Lett. B172 (1986) 412.
[23] M. Flato and C. Fronsdal, Lett. Math. Phys. 8 (1984) 159.

[24] B. Morel, A. Sciarrino and P. Sorba, Phys. Lett. B166 (1986) 69; erratum B167 (1986) 486.

[25] M.F. Sohnius, Nucl. Phys. B138 (1978) 109.

[26] M.F. Sohnius, Nucl. Phys. B136 (1978) 461.

[27] A. Galperin, E. Ivanov and V.I. Ogievetsky JETP Lett. 33 (1981) 168.

[28] J. Strathdee, Int. J. Math. Phys. 2 (1987) 273 and refs therein.

[29] M. Gunaydin and N. Marcus, Class. Quantum Grav. 2 (1985) L19.

[30] S. Ferrara, J. Wess and B. Zumino, Phys. Lett. B51 (1974) 239 